\title{
Usability Assessment of Two Different Control Modes for the Master Console of a Laparoscopic Surgical Robot
}

\author{
Xiaoli Zhang ${ }^{1}$, Carl A. Nelson ${ }^{2,3,4}$ and Dmitry Oleynikov ${ }^{3,4}$ \\ ${ }^{1}$ Division of Engineering and Physics, Wilkes University, Wilkes-Barre, PA USA \\ ${ }^{2}$ Department of Mechanical and Materials Engineering, University of Nebraska- \\ Lincoln (UNL), Lincoln, NE USA \\ ${ }^{3}$ Department of Surgery, University of Nebraska Medical Center (UNMC), Omaha, \\ NE USA \\ ${ }^{4}$ UNMC Center for Advanced Surgical Technology, Omaha, NE USA
}

Submitted March 2011. Accepted for publication December 2011.

\begin{abstract}
The objective of this study is to evaluate potential interface control modes for a compact fourdegree-of-freedom (4-DOF) surgical robot. The goal is to improve robot usability by incorporating a sophisticated haptics-capable interface. Two control modes were developed using a commercially available haptic joystick: (1) a virtually point-constrained interface providing an analog for constrained laparoscopic motion (3-DOF rotation and 1-DOF translation), and (2) an unconstrained Cartesian input interface mapping more directly to the surgical tool tip motions. Subjects $(n=5)$ successfully performed tissue identification and manipulation tasks in an animal model in point-constrained and unconstrained control modes, respectively, with speed roughly equal to that achieved in similar manual procedures, and without a steep learning curve. The robot control was evaluated through bench-top tests and a subsequent qualitative questionnaire $(\mathrm{n}=$ 15). The results suggest that the unconstrained control mode was preferred for both camera guidance and tool manipulations.
\end{abstract}

Keywords: minimally invasive surgery (MIS), computer-assisted surgery, human-robot interfaces, surgical robotics

\section{INTRODUCTION}

The use of current commercial robotic surgical systems $[1,2]$ is limited by their considerable size, complexity, and cost. For example, setting up the robotic approach for certain surgical approaches can be quite difficult [3], and the size and shape of the robot can also make access to the patient by personnel difficult. Some commercial robot 
systems with more limited applications such as endoscope guidance include ViKY [4] and LapMan [5]. Other examples of research systems include various kinematic architectures [6,7]. These systems typically have simple control interfaces such as 2degree-of-freedom (2-DOF) joysticks or foot pedals (in some cases vocal commands can be used). For example, a precursor to the ViKY system [8] used 4 of the 6 available DOF of a commercially available joystick as inputs. Another study proposed a combination of voice commands and image recognition using surgical task context for pointing a robotic laparoscopic camera [9]. Gaze tracking has also been proposed with proof-of-concept demonstration [10]. Other research systems have been developed for both characterizing and performing tasks associated with surgery, such as devices for measuring and characterizing tasks in laparoscopic surgery [11], systems for percutaneous needle insertion [12], and haptic systems for surgical interaction [13], among others.

Researchers continue to develop and test many different surgical robots in an attempt to improve the surgical robotics paradigm; of particular interest are robots for manipulating surgical tools in the paradigm of laparoscopy or minimally invasive surgery (MIS) using variations of spherical mechanisms to create remote centers of rotation collocated with the patient incision [14-16]. Each of these robotic systems has advantages and disadvantages. In this development, the human interface often receives less attention from designers in favor of the robot architecture itself. Very simple joysticks are often used to drive the robot's degrees of freedom. At the other extreme, customized high-fidelity haptic interfaces have been developed, generally for surgical simulation, to mimic direct handling of tools by surgeons [17-19]. Somewhere in the middle, there may exist effective and easy-to-use interfaces for controlling surgical robots. Although well designed control interfaces for some medical robot systems do exist, this topic of human-robot interfaces has not received its due attention in some cases, and interface design has not always been given the same level of emphasis as robot design. Some researchers have suggested guidelines for evaluation of the quality or effectiveness of interfaces. For example, voice commands, mouse control, and a mobile PDA-based mouse-like 2D interface were compared using an evaluation algorithm, resulting in a 1-6 effectiveness scale based on task accomplishment [20]. In a more recent work, a more general set of evaluation guidelines for haptic devices was proposed, and a common haptic device (presented in the next section) was found to have a high level of effectiveness (supporting its commercial popularity) [21].

A compact laparoscopic surgical robot prototype system was developed by the authors to reduce the size, weight, complexity and costs of robotic surgery systems and to simplify the setup procedure [22]. The robot, called CoBRASurge (Compact Bevelgeared Robot for Advanced Surgery), is a four-DOF robot for manipulation of laparoscopic cameras and surgical tools, and is characterized by its geared spherical mechanism which creates a remote center of motion coincident with the skin incision, mimicking the four-DOF motion typical of laparoscopic tool manipulation. As shown in Figure 1, the geared mechanism (links 2-7) allows the motors driving coaxial links 2,5 , and 6 to be placed remotely on a fixed base (link 1) rather than on the moving links of the robot. The forward and inverse kinematic derivation for this system is described in [23]. 


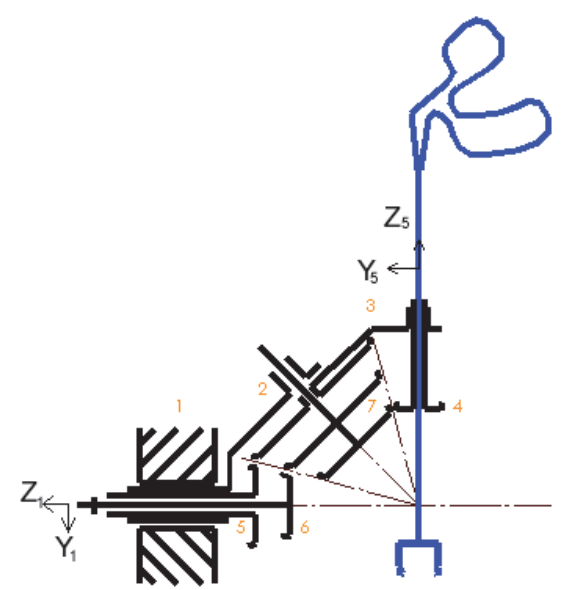

Figure 1. Kinematic structure of CoBRASurge robot. Base link 1, geared links 27, surgical tool held in link 4, with reference frames attached at ground (labeled frame 1) and on the tool (labeled frame 5).

The objective of the current study is to comparatively evaluate two user interface control modes for this compact single-port surgical robot with haptic (force feedback) and gravity compensation capabilities. Two interface control modes have been implemented in the system: (a) the virtually point-constrained joystick interface which was intended for camera guidance and mimics the feel of a fulcrum constraint at the incision point, and (b) the unconstrained, direct-position interface which was intended for surgical tool guidance and is more similar to control approaches used on some existing surgical robots [1, 24]. (Because we have also developed robotically actuated instruments, we consider both the cameras and instruments to be controllable using the robot.) Each interface is associated to a different mode of controlling the system. Although direct-position control interfaces have been used on surgical robots and accepted for over a decade [1], part of the motivation of this study was to determine whether they should necessarily be considered the preferred approach, considering the varying background and training of surgeons over time (as MIS has become more standard and incision-constrained surgical approaches are more emphasized) and different types of sub-tasks (pointing-based and manipulation-based) involved in MIS. Haptic capabilities and gravity compensation are available for both control modes to enhance robot functionality and control stability, if an interface device with haptic capability in the appropriate degrees of freedom is used. The goal is to make this robot more straightforward and easy to use by incorporating a more sophisticated, but not overly complex or expensive, haptic interface based on relatively ubiquitous hardware. Besides the advantage in ease of use, this prototype robotic surgical system is modular and compact, making telesurgical applications more practically feasible. This work describes efforts to evaluate usability of two interface designs for this robot. The authors are unaware of any other efforts to compare and evaluate different surgical robot control interface designs in this fashion. 


\section{METHODS AND PROCEDURES}

The kinematic derivation for the CoBRASurge robot allows mapping between motor position degrees of freedom and either input positions or input angles, each of these contributing to a different type of haptic input scheme. In the kinematic transforms [23], for the case where input angles are mapped to output angles, the following applies:

$$
\left[\begin{array}{l}
\theta_{21} \\
\theta_{51} \\
\theta_{61}
\end{array}\right]=\left[\begin{array}{ccc}
1 & 0 & 0 \\
1 & N_{35} & 0 \\
1 & N_{76} & N_{76} N_{47}
\end{array}\right]\left[\begin{array}{l}
* \theta_{21} \\
\theta_{32} \\
\theta_{43}
\end{array}\right]
$$

where the left-hand side constitutes the input angles, the column vector on the right contains the output angles of the basic serial spherical linkage which influence pitch, yaw, and roll, and $\mathrm{N}_{\mathrm{ij}}$ are gear ratios from the bevel gearing that couples the motion of the spherical linkage to the input axes. The pitch and yaw angles of the tool (rotation left/right and in/out of the page in Figure 1) are defined by the orientation of the Z-axis of reference frame 5 at the tool, expressed in the coordinates of the fixed global frame 1 at the robot base, as shown in Figure 1, and the roll is likewise identified from the orientation of the $\mathrm{X}$-axis of frame 5 .

For a desired pitch/yaw/roll tool orientation, the angles can be solved:

$$
\begin{aligned}
& * \theta_{21}=\theta_{21}=\tan ^{-1} \frac{{ }_{5}^{1} Z_{x}\left(c \theta_{32} c \alpha_{2} s \alpha_{3}+c \alpha_{3} s \alpha_{2}\right)+{ }_{5}^{1} Z_{y} s \theta_{32} s \alpha_{3}}{{ }_{5}^{1} Z_{x} s \theta_{32} s \alpha_{3}-{ }_{5}^{1} Z_{y}\left(c \theta_{32} c \alpha_{2} s \alpha_{3}+c \alpha_{3} s \alpha_{2}\right)} \\
& \theta_{32}=\tan ^{-1} \frac{ \pm \sqrt{1-\left(\frac{c \alpha_{3} c \alpha_{2}-{ }_{5}^{1} Z_{z}}{c \alpha_{3} c \alpha_{2}}\right)^{2}}}{\frac{c \alpha_{3} c \alpha_{2}-{ }_{5}^{1} Z_{z}}{c \alpha_{3} c \alpha_{2}}}=\tan ^{-1} \frac{ \pm \sqrt{s^{2} \alpha_{3} s^{2} \alpha_{2}-\left(c \alpha_{3} c \alpha_{2}-{ }_{5}^{1} Z_{z}\right)^{2}}}{c \alpha_{3} c \alpha_{2}-{ }_{5}^{1} Z_{z}} \\
& ' \theta_{43}=\tan ^{-1}\left[\frac{{ }_{5}^{1} X_{y}\left(c \theta_{32} c \theta_{21}-s \theta_{32} s \theta_{21} c \alpha_{2}\right)-{ }_{5}^{1} X_{x}\left(c \theta_{32} s \theta_{21}+s \theta_{32} c \theta_{21} c \alpha s \alpha_{3}\right)}{{ }_{5}^{1} X_{x}\left(c \theta_{32} c \theta_{21} c \alpha_{2} c \alpha_{s}-s \theta_{32} s \theta_{21} c \alpha_{3}-c \theta_{21} s \alpha_{2} s \alpha_{3}\right)-{ }_{5}^{1} X_{y}\left(-c \theta_{32} s \theta_{21} c \alpha_{2} c \alpha_{s}-s \theta_{32} c \theta_{21} c \alpha_{3}+s \theta_{21} s \alpha_{2} s \alpha_{3}\right)}\right]
\end{aligned}
$$

where ${ }_{5}^{1} Z_{x[y, z]}$ is the projection of the Z-axis of reference frame 5 on the X[Y,Z]-axis of frame $1, \mathrm{c}$ and $\mathrm{s}$ represent sine and cosine, and $\alpha_{\mathrm{i}}$ are parameters representing the angular geometry of the bevel gearing. The input angles are then calculated using eqn. 1. For the case where input positions are mapped to output angles, defining a vector from the desired $x-y-z$ position to the fixed rotation center of the mechanism generates the required vector ${ }_{5}^{1} Z$, and the length of that vector gives the penetration depth (the $4^{\text {th }}$ degree of freedom of the robot). 
The manual input interface consists of a Phantom Omni (SensAble) haptic joystick [25] which is able to provide three DOF of position input information in Cartesian space, three DOF of rotation input, and three DOF of (Cartesian) force output to the user. A laptop computer running LabVIEW software (National Instruments) was used to create a flexible, modular user interface for the controls, including motion scaling. The joystick feedback runs at $1000 \mathrm{~Hz}$, and the motor driver hardware runs at 500 to $2000 \mathrm{~Hz}$, with the kinematic calculations refreshing at $120 \mathrm{~Hz}$. The selection of a master-slave scaling ratio can affect the motion quality (accuracy, velocity, and smoothness of motion trajectories), task completion time, and the master's motion range. Surgeons can scale up/down the motion ratio depending on the accuracy/sensitivity requirement of tasks and personal preference.

\subsection{Setup and Control Modes}

Two different types of control interface were evaluated using the robot. Although some joystick devices are spring-loaded to return to a "home" position, the input devices employed in this study do not have such a default position; therefore, instead of a "jog" velocity control, both control modes implement position control. This is more representative of how a surgical assistant would typically orient a laparoscopic camera, which was part of the initial rationale of the study. The details and implementation of the two control modes, along with the hardware configuration for setting up experiments on the robot, are described in the following subsections.

\subsubsection{Virtual Point Constraint Method}

In the virtually point-constrained scheme (Figure 2), "virtual constraints" were programmed using the haptic features of the joystick in such a way that the tip of the joystick stylus is fixed at a point in space (using stiff virtual springs). This leaves the three rotational DOF (indicated by yellow arrow lines in Figure 2) free (directly mapped using the above equations), and the fourth translational DOF is mapped to buttons on the stylus (under the index finger in Figure 2). This control approach is similar to that employed in [26] in the sense that the joystick's rotational DOFs are used to command the tool orientation, although no virtual constraint was created in that case and the penetration depth was controlled differently (using position coordinates of the stylus tip). The rationale for creating this control mode has to do with the difference between pointing tasks and fine manipulation tasks. When there is a fulcrum effect, pointing at an object is felt more natural than object manipulation; similar configurations for aiming are found in daily life such as in tripod-mounted telescopes. The virtually pointconstrained joystick interface provides an analog for incision-constrained laparoscopic motion. Since the Phantom Omni only has haptic capabilities in the Cartesian position space and not the rotation space, this leaves out the possibility of force feedback in this mode. However, the same manufacturer provides a Premier model with full 6-DOF haptics which could be used to restore this functionality (at significant additional cost). Alternatively, other haptic devices such as those developed as standalone simulators (e.g., Immersion [17]) could potentially be co-opted for use. 


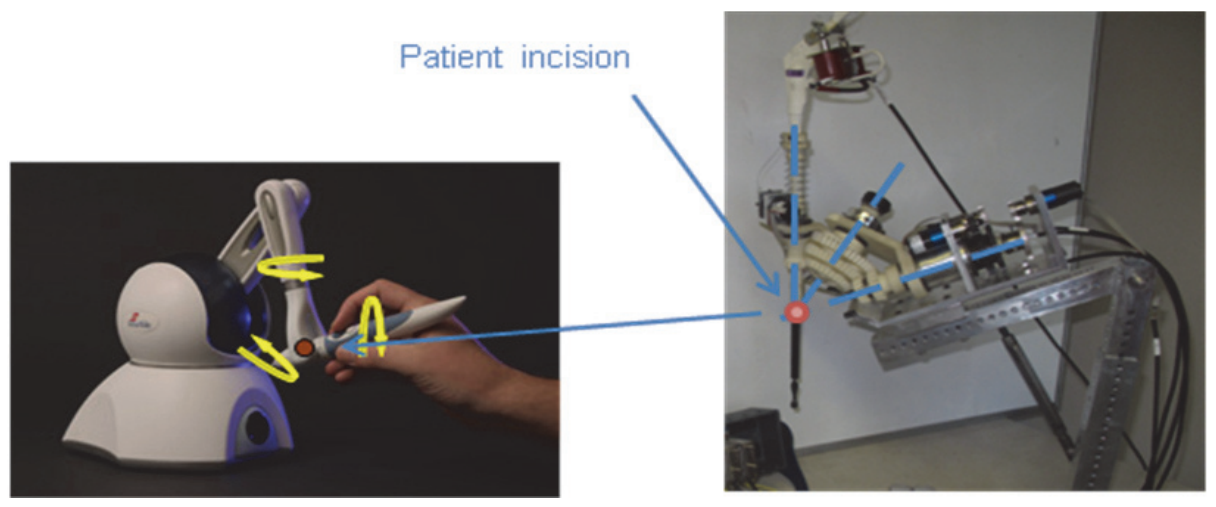

Figure 2. The virtually point-constrained control scheme. Arrows show rotations about a fixed point on the joystick (left). Rotation axes (broken blue lines) coincide at a corresponding point on the manipulator (right).

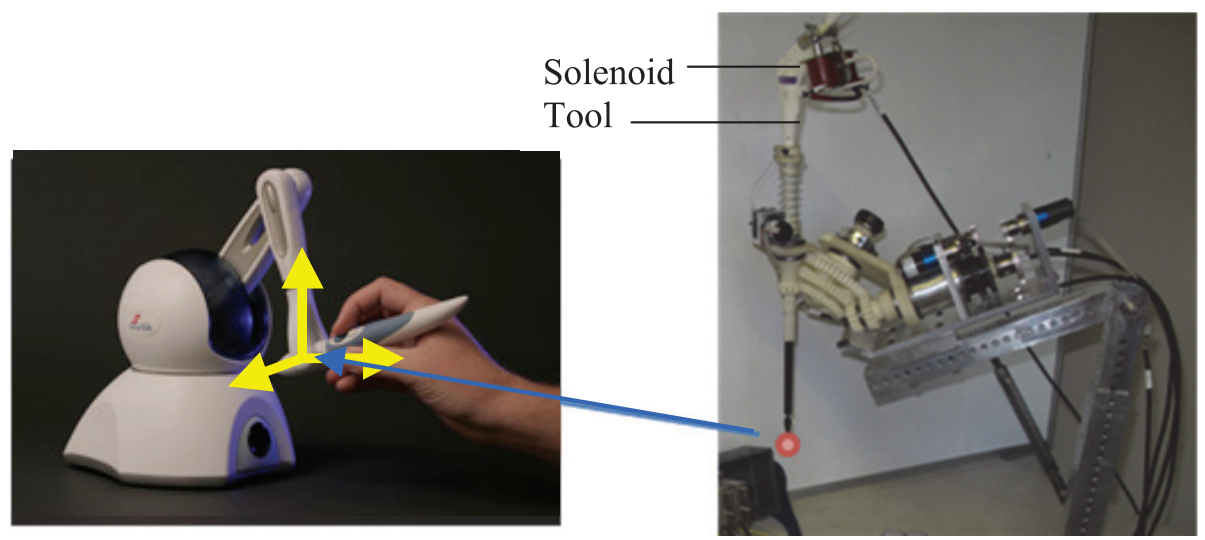

Figure 3. The unconstrained, direct-position control scheme. The stylus tip, shown at the origin of the local moving coordinate frame of the joystick (left), is mapped to the tip of the surgical tool held in the manipulator (right).

\subsubsection{Cartesian Input Method}

In addition to the "virtual constraint" paradigm designed for camera manipulation, a modified kinematic control scheme was also implemented for surgical tool guidance. In this scheme (shown in Figure 3), the tip of the tool is mapped to the endpoint of the joystick stylus (indicated by the blue arrow in Figure 3) as described above (three translational DOF in Cartesian space are used rather than three rotational DOF in rotation space) in order to achieve a feel more similar to open surgery (unconstrained six-DOF motion), although the tool is still constrained by its insertion through the trocar. For initialization, a central point in the robot workspace is mapped to a central point in the joystick workspace; motion scaling is adjustable in the software to either 
reach more robot workspace or fine-tune for precise motion. The kinematics can be checked at each iteration of the position control loop to disallow joystick commands which lie outside the robot workspace. Haptic functionality with three-DOF force output is enabled or disabled by the click of a button on the stylus to provide the motion constraints for convenience of using the joystick. This toggling feature is quite convenient if the surgeon needs to hold the tool stationary during surgical procedures as it prevents accidental or gravity-induced motions of the joystick stylus and the robotic tool, since the joystick itself is not gravity-compensated (statically balanced). This control mode is very similar to those used in other existing surgical robots [8, 24], except that the kinematic mapping is different. In concept, it is also similar to the input devices of the da Vinci Surgical System [1] and Sensei X guided catheter system [27], as these also map Cartesian input motion to the endpoint motion of the surgical instrument, although the input devices in these cases are specially designed and integrated system components. Generally speaking, tool-tissue interactions can be mapped from the tool tip back through to the joystick stylus, given an appropriate model (as discussed further below).

\subsubsection{Tool Control Hardware}

A simple solenoid-actuated surgical tool was combined with the robot for tissue grasping in animal experiments to be described below, as shown in Figure 3. The control button for the solenoid actuation (not visible in Figure 3) is a simple off-theshelf switch temporarily strapped to the handle of the joystick stylus such that all robot functionality is available in one-handed control. (This could also be mapped to one of the existing stylus buttons if desired.)

As shown in Figure 4, the robot actuators and laptop are interfaced with a proportional-integral-derivative (PID) control algorithm using a field-programmable CompactRIO system (National Instruments) for better flexibility, robustness, and compactness of the hardware system. The modularity of this hardware allows not only for robot motor control but also for sensing of motor effort. Inverse dynamic equations as described in [28] can be programmed in LabVIEW to infer the forces applied at the tool tip (sensorless haptics using motor effort only as the feedback signal), and this can be used to provide more accurate force feedback at the joystick. If the pose of the robot is known in the global reference frame, the portion of motor effort which is attributable to the self-weight of the robotic linkage can also be determined using the information contained in the Jacobian. Having an accurate kinematic and dynamic model is necessary in order to implement torque compensation for removing gravitational effects due to the weight of the robot and/or dynamic loads due to D'Alembert (inertial) effects from the joystick-side feedback if it is used to represent tool-tissue interactions. With this compensation, the fidelity of the feel of the controller can be increased such that the haptic joystick feedback corresponds primarily to tissue interaction forces. In this sense, the gravity compensation can be considered an extension or enhancement of haptic feedback. Note that this is different from implementing a statically balanced, clutched, or otherwise stationary input device, and that friction effects at the trocar would still be felt at the haptic input device. 


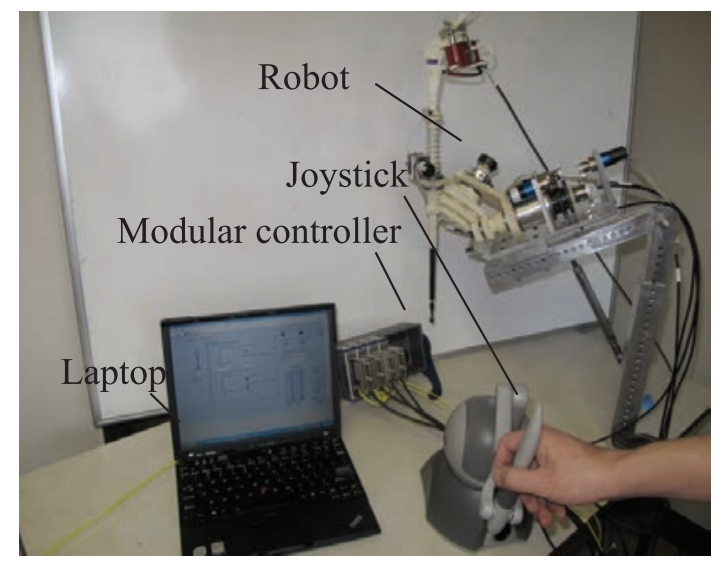

Figure 4. The control hardware system including the laptop, the motion controller, the joystick, and the surgical robot.

\subsection{Bench-Top and Clinical Testing}

Bench-top testing was performed for both control modes to evaluate the usability of the user interfaces for camera guidance (point-constrained, shown in Figure 5) and tool manipulation (Cartesian). Fifteen subjects (three with and twelve without laparoscopic surgical experience, comprising surgeons, medical students, and engineering students) participated in the bench-top tests in sessions lasting approximately 5 minutes. Haptic features including gravity compensation were disabled in all tests so that the pointconstrained and Cartesian control modes could be compared on an equal basis. The benchtop task was performed using custom master-slave scaling ratios based on each subject's preference as indicated at the beginning of the session (within a reasonable range, with the maximum scaling no greater than double the minimum scaling).

Animal experiments on porcine models were performed in a separate facility by five subjects (two with and three without laparoscopic experience) as follows. In all cases, regulations of the International Committee of Medical Journal Editors concerning protection of human subjects and animals were followed.

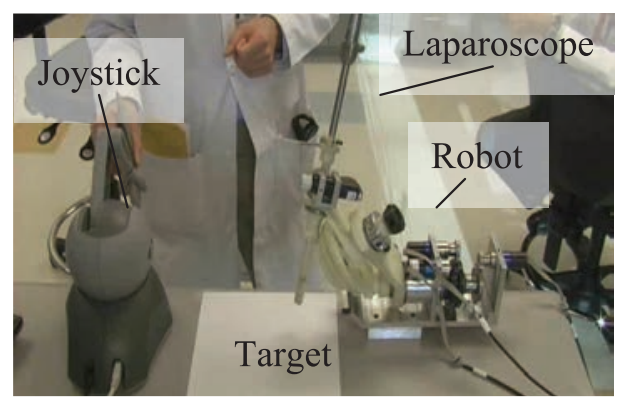

Figure 5. Bench-top test using the surgical robot for camera guidance. 


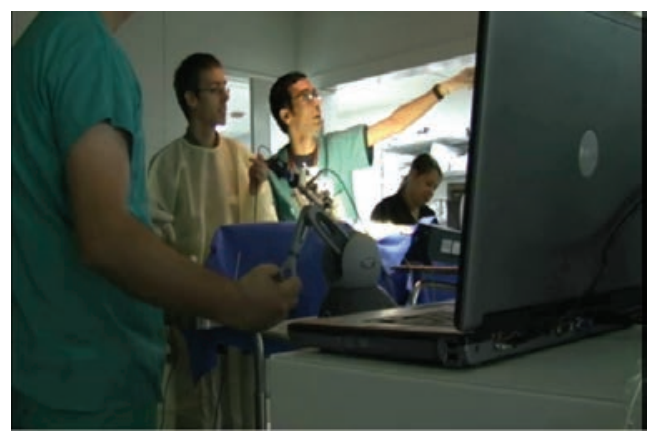

Figure 6. Animal test using the surgical robot for camera guidance.

\subsubsection{Diagnostic Laparoscopy}

The target in this experiment is to guide a laparoscopic camera to identify five organs including bowel, stomach, spleen, liver, and gall bladder, in random order, in a single animal model. The robot was mounted to the side railing of a standard surgical table using a custom-built support arm, such that its remote center of rotation coincided with the planned incision point. A single trocar was placed, and the laparoscopic camera was then inserted through the trocar into the abdomen and fixed to the robot. Subjects then used video feedback obtained by the robot-mounted camera to identify the anatomical features. The general layout is shown in Figure 6, with the subject operating the joystick in the foreground next to the control computer, and the robot mounted over the animal in the background; the video display is outside the upper right corner of the image.

\subsubsection{Tool Manipulation}

The goal in this experiment is to use the solenoid-actuated surgical tool described in Section 2.1.3 to grasp tissues in the animal model as instructed by a surgeon. The surgical tool was inserted through the trocar into the abdomen and fixed to the robot (as in the diagnostic laparoscopy experiment). A view of the surgical field was obtained through an endoscope controlled manually by a surgical assistant through a separately placed trocar, and presented in the video monitor. Subjects were instructed to find and then grasp or manipulate the target anatomical features using the robotically controlled tool. Figure 7 shows the general layout, with the joystick and operator in the foreground, the robot mounted over the animal with the grasper inserted, and the video monitor behind. Laparoscope video and external digital video were both recorded during the experiments. 


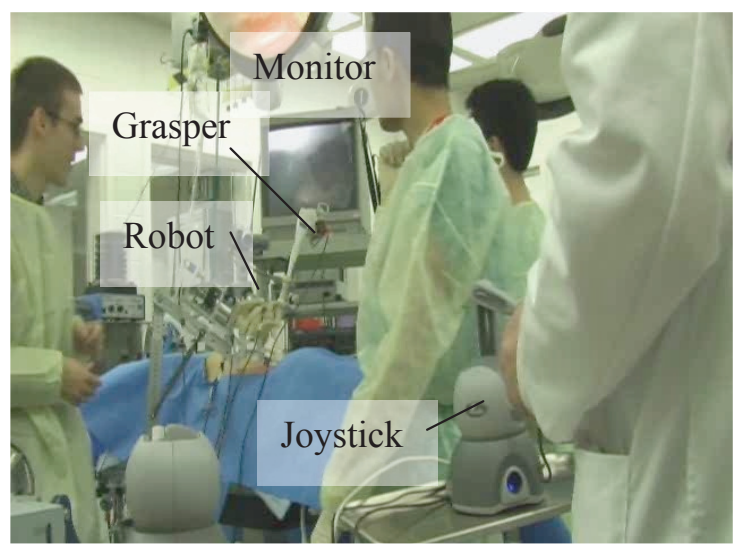

Figure 7. Animal tests using the surgical robot for tool manipulation.

\subsubsection{Benchtop Testing and Questionnaire}

Using the same mounting arm as in the animal tests, the robot was suspended above a worktable marked with visual reference points, and a laparoscope was fitted in the robot. All fifteen subjects (comprising surgeons, medical students, and engineering students) were surveyed to compare the two control modes in this environment by navigating the robot at will over a period of several minutes. After consenting to participation, each subject was given time to practice with each of the control modes and set their preferred motion scaling, until they are ready to complete the questionnaire.

The questionnaire consists of two sections (see APPENDIX). The first portion (Section A) of the questionnaire was only for the subjects with laparoscopic surgery experience to compare the effectiveness of the robot against the baseline of manual laparoscopic surgery. In addition to the 3 subjects with laparoscopic experience who participated in the benchtop experiment, 2 others who took part in the animal experiment completed this section based on that exposure to the robotic system, making a total of 5 respondents. In the second section of the questionnaire, the subjects were asked to evaluate if each of the control modes was well suited for camera guidance and tool manipulation, respectively. The subjects were also asked to evaluate (a) how stable/smooth/easy each control mode seemed to be for camera guidance and tool manipulation, respectively, (b) the motion scaling feature, (c) intention to use the tool if commercially available, and (d) preference of control mode. Although haptics were disabled for these tests as mentioned above, these features were explained to the subjects, and they were asked if haptic feedback for sensing tool-tissue forces would be considered helpful, and if the gravity compensation feature would be helpful. The responses to all questions were statistically analyzed. 


\section{RESULTS}

Bench-top and clinical animal testing confirmed qualitatively that the robot control is stable and smooth. Using a single animal model, all five subjects were able to correctly identify five anatomical targets in random order, and then manipulate tissues as directed by an expert, in the two respective control modes (first the constrained and then the unconstrained mode), with speed roughly equal to that achieved in similar manual procedures. (Although specific times to accomplish the tasks were not measured, this statement is based on comments from participants with laparoscopic experience.) It should be noted that this good result was obtained despite users having little experience either with the robot or the task.

Table 1. Experienced users' feedback on comparison between manual and robotic tools (significance based on t-test); responses converted to 5-point scale $(1$ = very bad, 3 = indifferent, 5 = very good $)$.

\begin{tabular}{|c|c|c|c|c|c|c|c|c|}
\hline & \multicolumn{2}{|c|}{ Comfort } & \multicolumn{2}{c|}{ Smoothness } & \multicolumn{2}{c|}{ Stability } & \multicolumn{2}{c|}{ Accuracy } \\
\hline & Manual & Robotic & Manual & Robotic & Manual & Robotic & Manual & Robotic \\
\hline Mean & 3.60 & 3.00 & 3.40 & 2.60 & 2.80 & 4.00 & 3.00 & 3.20 \\
\hline $\begin{array}{c}\text { Standard } \\
\text { deviation }\end{array}$ & 0.80 & 0.63 & 0.49 & 0.49 & 0.75 & 0.00 & 0.63 & 0.75 \\
\hline Significance & \multicolumn{2}{|c|}{ No $(\mathrm{p}=0.273)$} & \multicolumn{2}{|c|}{ Yes $(\mathrm{p}=0.050)$} & \multicolumn{2}{c|}{ Yes $(\mathrm{p}=0.012)$} & \multicolumn{2}{c|}{ No $(\mathrm{p}=0.694)$} \\
\hline
\end{tabular}

In the first section of the questionnaire requiring surgical manipulation experience (see Table 1), $60 \%$ of surgeons (3 of 5 participants completing this section) indicated no difference between the robotic manipulation and manual manipulation in comfort and smoothness. Although the overall response indicated that robotic manipulation was less smooth, this could reflect a perception on the part of some respondents that manual manipulation is "perfectly smooth." All of these respondents evaluated the robotic manipulation as having a higher stability compared to manual manipulation. In the second section (all 15 participants responding), the results (shown in Figure 8(a,b)) suggest that the unconstrained control mode was preferred for both camera guidance and tool manipulation. Respondents indicated that the unconstrained interface gives a feel more like the direct control in open surgery. Subjects' responses to the described potential for gravity compensation and force feedback were positive; the average response indicated that users believed gravity compensation would slightly improve the ability to perform surgical tasks. Eighty percent (80\%) of the subjects indicated that they would be interested in using the surgical robot once commercially available. 


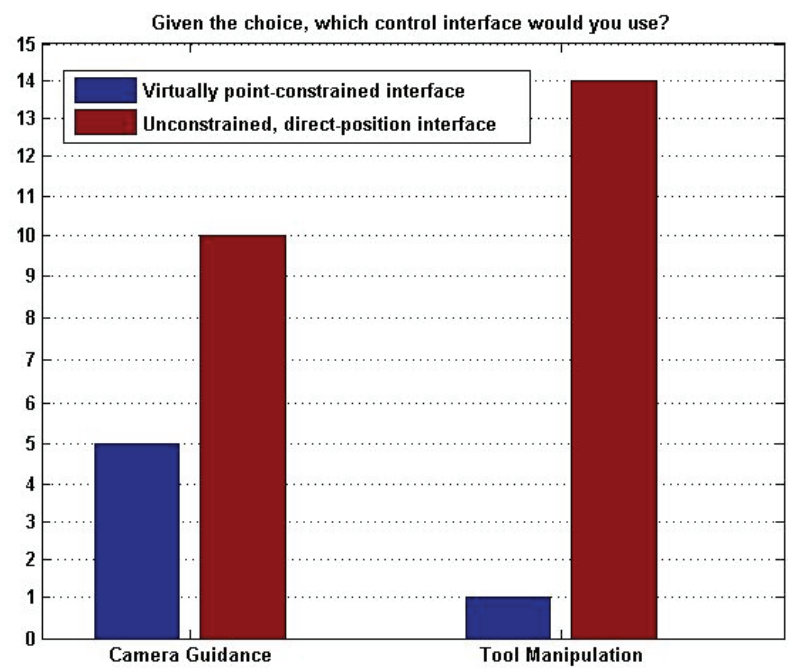

(a)

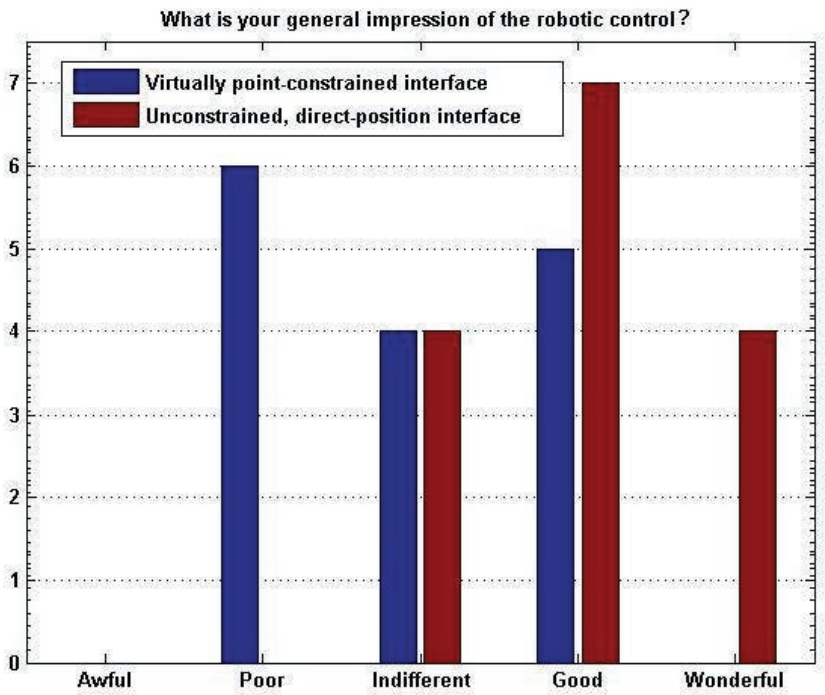

(b)

Figure 8. Questionnaire outcomes $(\mathrm{n}=15)$. Number of responses indicated on the $y$-axis. (a) User preferences of two control modes for camera guidance and tool manipulation: sign test shows significant preference for unconstrained mode during tool manipulation $(\mathrm{p}=0.001)$ but is inconclusive for camera guidance tasks $(\mathrm{p}=0.118)$. (b) Respondents' general impressions of two control modes: t-test shows significant preference for unconstrained mode $(\mathrm{p}=0.001)$. 


\section{DISCUSSION}

The statistical results show that subjects see benefits of the surgical robotic system with an appropriately designed and configured control interface. Most subjects responded that the surgical robot system can provide more stable manipulation, as shown by the significance results in Table 1, which could mitigate problems experienced during manual surgery. The unconstrained control mode was preferred for both camera guidance and tool manipulation (see Figure 8), possibly because it provides motion more similar to open surgery and more natural to the surgeon who does not have the perception of incision-constrained motion as in manual laparoscopy, not being collocated with the patient but rather operating through a remote interface. This explanation merits further investigation to verify quantitatively.

Another factor which may accentuate the significant preference of the unconstrained control mode for both camera guidance and tool manipulation could be the degree of subjects' experience with manual laparoscopic surgery. Individuals without laparoscopic experience might prefer the unconstrained control mode more than those with laparoscopic experience. The respondents' comments suggest that subjects without incision-constrained motion training typical for manual laparoscopy might find the lack of pivot constraints more natural and adjust to this interface more quickly. In this study, the majority of subjects do not have experience in laparoscopic surgery. Further quantitative tests are needed to verify the current questionnaire result for subjects at different skill levels.

In addition, subjects' response to the toggling feature for holding the tool stationary during surgical procedures was overwhelmingly positive. The qualitative responses also indicate that the surgeons believe the functionality of motion scaling, gravity compensation, and force feedback will greatly improve the performance of the surgical robot. Test results showed that the performance of this robotic surgery system and interface improves upon manual instrument operation in terms of motion stability and possibly in terms of accuracy (see Table 1) yet without a steep learning curve (based on very little practice with the robotic system) or a significant increase in time for task completion. Future studies should include more quantitative and statistical evaluations involving metrics such as task performance time and tool travel distances [29-33] to determine if users' perceptions of control mode effectiveness are supported by measured efficiencies.

There remain some limitations of this system. The solenoid-actuated surgical tool was able to grasp tissues, but the solenoids should be replaced with continuous rather than discrete actuators to eliminate vibration effects in the tool due to sudden discontinuous motions. The range of motion of the joysticks is somewhat limited, which in turn limits the level of motion scaling that can be practically and efficiently implemented. In the unconstrained control mode, the joystick tip position is the primary input, but its orientation is not relevant to the tool output motion. This could be confusing to some users, but could be alleviated by integration with a fully (6-DOF) haptic joystick to allow the joystick orientations to be constrained through software. As indicated in Table 1, smoothness and comfort of the system should be improved in future design revisions in order to encourage adoption and enhance usability. Despite these limitations, overall, the current results suggest that the current system and its proposed control modes constitute a step forward in surgical robotics applications. 


\section{CONCLUSION}

Two control modes for a compact single-port surgical robot manipulator were developed and their effectiveness evaluated. A main advantage of the interface is the use of relatively easy-to-use, off-the-shelf, haptics-enabled joysticks. Results from clinical experiments and feedback in benchtop settings were positive, and indicated that the preferred method of employing the joysticks is through unconstrained mapping of joystick motions to surgical tool tip motions, rather than mimicking surgical tool kinematic constraints using the haptic features of the joysticks. The next step to take in the development of the highly autonomous surgical robot system is to integrate with multifunction robotic surgical end effectors [34], and develop a more comfortable button mapping in the master joystick handle to control the robot easily and precisely. Also, to improve the performance of the interfaces, new experiments are planned including more advanced surgical procedures. Quantitative comparative evaluations, including comparisons to other existing surgical systems, are needed to build upon the current findings and to identify directions for further improvement.

\section{CONFLICT OF INTEREST: None.}

\section{NOMENCLATURE}

$\mathrm{N}_{\mathrm{ij}} \quad$ gear ratio corresponding to gear sizes on links $\mathrm{i}$ and $\mathrm{j}$

${ }_{5}^{1} X[Y, Z]_{x[y, z]} \quad$ projection of the X[Y,Z]-axis of frame 5 (robot end effector) on the $\mathrm{x}[\mathrm{y}, \mathrm{z}]$-axis of frame 1 (ground)

\section{Greek}

\section{REFERENCES}

[1] Intuitive Surgical, Inc. da Vinci Surgical System. http://www.intuitivesurgical.com. Accessed November 2011

[2] Guthart GS, Salisbury JK. The Intuitive Telesurgery System: Overview and Application. Proc. IEEE International Conference on Robotics and Automation. 2000; 1:618-621.

[3] Song HJ. The Docking of Robotic Arms in Robot-Assisted Endoscopic Thyroidectomy. Proc. MIRA $4^{\text {th }}$ International Congress. 2009.

[4] EndoControl. EndoControl: ViKY Endoscope Positioner. http://www.endocontrolmedical.com/viky_ep.php. Accessed February 12, 2011.

[5] LapMan. LapMan. http://www.lapman.ch/robots.php. Accessed February 12, 2011.

[6] Thielmann S, Seibold U, Haslinger R, Passig G, Bahls T, Jörg S, Nickl M, Nothhelfer A, Hagn U, Hirzinger G. MICA - A New Generation of Versatile Instruments in Robotic Surgery. Proc. IEEE/RSJ International Conference on Intelligent Robots and Systems. 2010; 1:871-878.

[7] Li F, Hu L, Kuang S, Yang C, Wang T. A 5-DOF Table-Mounted Surgical Robot. Proc. IEEE International Conference on Robotics and Biomimetics. 2007; 1:355-359.

[8] Ma J, Berkelman P. Control Software Design of a Compact Laparoscopic Surgical Robot System. Proc. IEEE International Conference on Intelligent Robots and Systems. 2006; 1:2345-2350.

[9] Ko SY, Kwon DS. A Surgical Knowledge Based Interaction Method for a Laparoscopic Assistant Robot. Proc. IEEE International Workshop on Robot and Human Interactive Communication. 2004; $1: 313-318$ 
[10] Noonan DP, Mylonas GP, Shang J, Payne CJ, Darzi A, Yang GZ. Gaze Contingent Control for an Articulated Mechatronic Laparoscope. Proc. 3rd IEEE RAS and EMBS International Conference on Biomedical Robotics and Biomechatronics. 2010; 1:759-764.

[11] Rosen J, Brown JD, Barreca M, Chang L, Hannaford B, Sinanan M. The Blue DRAGON - A System for Monitoring the Kinematics and Dynamics of Endoscopic Tools in Minimally Invasive Surgery for Objective Laparoscopic Skill Assessment. Proc. Medicine Meets Virtual Reality, 2002. Studies in Health Technology and Informatics. 85:412-418.

[12] Cleary K, Watson V, Lindisch D, Stoianovici D, Patriciu A, Mazilu D, Taylor RH, Fichtinger G, Xu S, White CS, Donlon J, Taylor M. Precision Placement of Instruments for Minimally Invasive Procedures using a 'Needle Driver' Robot. International Journal of Medical Robotics and Computer Assisted Surgery. 2005; 1(2):1-9.

[13] Sabater JM, Garcia N, Pérez C, Azorin JM. Algorithm for Haptic Rendering of Reconstructed 3D Solid Organs. International Journal of Computer Assisted Radiology and Surgery. 2008; 3:387-393.

[14] Kim SK, Shin WH, Ko SY, Kim J, Kwon DS. Design of a Compact 5-DOF Surgical Robot of a Spherical Mechanism: CURES. Proc. IEEE/ASME International Conference on Advanced Intelligent Mechatronics. 2008; 1:990-995.

[15] Zemiti N, Ortmaier T, Morel G. A New Robot for Force Control in Minimally Invasive Surgery. Proc. IEEE Conference on Intelligent Robots and Systems. 2004; 4:3643-3648.

[16] Lum M, Friedman D, Rosen J, Sankaranarayanan G, King H, Fodero K, Leuschke R, Sinanan M, Hannaford B. The RAVEN - Design and Validation of a Telesurgery System. International Journal of Robotics Research, 2009; 28(9):1183-1197.

[17] Immersion. Featured Haptic Products for Medical. http://www.immersion.com/markets/medical/products/index.html. Accessed February 15, 2011.

[18] Simulab. LapTrainer with SimuVision. http://www.simulab.com/product/surgery/laparoscopic/laptrainer-simuvision. Accessed February 15, 2011.

[19] GMV. InsightArthro. http://insightarthrovr.gmv.com/index_en.htm. Accessed February 15, 2011.

[20] Fernández-Lozano J, Gómez-de-Gabriel JM, Muñoz VF, García-Morales I, Melgar D, Vara C, GarcíaCerezo A. Human-Machine Interface Evaluation in a Computer Assisted Surgical System. Proc. IEEE International Conference on Robotics and Automation. 2004; 1:231-236.

[21] Andonovski B, Ponsa P, Casals A. Towards the Development of a Haptics Guideline in Human-Robot Systems. Proc. 3rd International Conference on Human System Interaction. 2010; 1:380-387.

[22] Zhang X, Oleynikov D, Nelson CA. Portable Tool Positioning Robot for Telesurgery. Proc. Medicine Meets Virtual Reality 17, 2009. Studies in Health Technology and Informatics. 142:438-443.

[23] Zhang X, Nelson CA. Kinematic Analysis and Optimization of a Novel Robot for Surgical Tool Manipulation. ASME Journal of Medical Devices. 2008; 2(2):021003.1-021003.8.

[24] Sankaranarayanan G, King H, Ko SY, Lum MJH, Friedman DCW, Rosen J, Hannaford B. Portable Surgery Master Station for Mobile Robotic Telesurgery. Proc. ROBOCOMM. 2007.

[25] Massie TH, Salisbury JK. The Phantom Haptic Interface: A Device for Probing Virtual Objects. Proc. ASME Winter Annual Meeting, Symposium on Haptic Interfaces for Virtual Environment and Teleoperator Systems. 2005; 55(1):295-299.

[26] Kobayashi Y, Tomono Y, Sekiguchi Y, Watanabe H, Toyoda K, Konishi K, Tomikawa M, Ieiri S, Tanoue K, Hashizume M, Fujie MG. A Surgical Robot with Vision Field Control for Single Port Endoscopic Surgery. The International Journal of Medical Robotics and Computer Assisted Surgery. 2010; 6:454-464.

[27] Hansen Medical. Sensei X Robotic Catheter System. http://www.hansenmedical.com/sensei. Accessed July 12, 2011.

[28] Zhang X, Nelson CA. On the Dynamic Modeling of a Bevel-Geared Surgical Robotic Mechanism. ASME Journal of Medical Devices. 2010; 4(4): 041002.1-041002.11.

[29] Satava R, Cuschieri A, Hamdorf J. Metrics for Objective Assessment. Surgical Endoscopy. 2003; 17(2):220-226. 
[30] Huang J, Payandeh S, Doris P, Hajshirmohammadi I. Fuzzy Classification: Towards Evaluating Performance on a Surgical Simulator. Proc. Medicine Meets Virtual Reality 13, 2005. Studies in Health Technology and Informatics. 111:194-200.

[31] Nebot PB, Jain Y, Haylett K, et al. Comparison of Task Performance of the Camera-Holder Robots EndoAssist and Aesop. Surg Laparosc Endosc Percutan Techn. 2003; 13(5):334-338.

[32] Yavuz Y, Ystgaard B, Skogvoll E, et al. A Comparative Experimental Study Evaluating the Performance of Surgical Robots Aesop and Endosista. Surg Laparosc Endosc Percutan Techn. 2000; 10(3):163-167.

[33] Derossis AM, Fried GM, Abrahamowicz M, Sigman HH, Barkun JS, Meakins JL. Development of a Model for Training and Evaluation of Laparoscopic Skills. The American Journal of Surgery. 1998; 175(6):482-487.

[34] Nelson CA, Miller DJ, Oleynikov D. Design Methodology for a Novel Multifunction Laparoscopic Tool: Engineering for Surgeons' Needs. Proc. Medicine Meets Virtual Reality 15, 2007. Studies in Health Technology and Informatics. 125:343-348. 


\section{APPENDIX}

\section{Questionnaire sample}

Section A. Subjective evaluation of the manual manipulation and robotic manipulation of laparoscopic cameras

How many years have you been performing laparoscopic surgery?

1. How comfortable is the manual manipulation and robotic manipulation of laparoscopic camera?

\begin{tabular}{|c|c|}
\hline Manual manipulation of camera & Robotic manipulation of camera \\
\hline$\square$ Extremely uncomfortable & $\square$ Extremely uncomfortable \\
$\square$ Uncomfortable & $\square$ Uncomfortable \\
$\square$ Indifferent & $\square$ Indifferent \\
$\square$ Comfortable & $\square$ Comfortable \\
$\square$ Extremely comfortable & $\square$ Extremely comfortable \\
\hline
\end{tabular}

2. How smooth is the manual manipulation and robotic manipulation of laparoscopic camera?

\begin{tabular}{|l|l|}
\hline Manual manipulation of camera & Robotic manipulation of camera \\
\hline$\square$ Extremely unsmooth & $\square$ Extremely unsmooth \\
$\square$ Unsmooth & $\square$ Unsmooth \\
$\square$ Indifferent & $\square$ Indifferent \\
$\square$ Smooth & $\square$ Smooth \\
$\square$ Extremely smooth & $\square$ Extremely smooth \\
\hline
\end{tabular}

3. How stable is the manual manipulation and robotic manipulation of laparoscopic camera?

\begin{tabular}{|l|c|}
\hline Manual manipulation of camera & Robotic manipulation of camera \\
\hline$\square$ Extremely unstable & $\square$ Extremely unsmooth \\
$\square$ Un stable & $\square$ Unsmooth \\
$\square$ Indifferent & $\square$ Indifferent \\
$\square$ Stable & $\square$ Smooth \\
$\square$ Extremely stable & $\square$ Extremely Smooth \\
\hline
\end{tabular}

4. How accurate is the manual manipulation and robotic manipulation of laparoscopic camera?

\begin{tabular}{|l|c|}
\hline Manual manipulation of camera & Robotic manipulation of camera \\
\hline$\square$ Extremely unaccurate & $\square$ Extremely unaccurate \\
$\square$ Unaccurate & $\square$ Unaccurate \\
$\square$ Indifferent & $\square$ Indifferent \\
$\square$ Accurate & $\square$ Accurate \\
$\square$ Extremely accurate & $\square$ Extremely accurate \\
\hline
\end{tabular}




\section{Section B. Subjective evaluation of robotic interfaces}

1. How easy is the robotic manipulation?

\begin{tabular}{|l|l|l|l|}
\hline \multicolumn{2}{|l|}{ Virtually point-constrained joystick interface } & \multicolumn{3}{|l|}{ Unconstrained, direct-position interface } \\
\hline Camera Guidance & Tool Manipulation & Camera Guidance & Tool Manipulation \\
\hline$\square$ Extremely difficult & $\square$ Extremely difficult & $\square$ Extremely difficult & $\square$ Extremely difficult \\
$\square$ Difficult & $\square$ Difficult & $\square$ Difficult & $\square$ Difficult \\
$\square$ Indifferent & $\square$ Indifferent & $\square$ Indifferent & $\square$ Indifferent \\
$\square$ Easy & $\square$ Easy & $\square$ Easy & $\square$ Easy \\
$\square$ Extremely easy & $\square$ Extremely easy & $\square$ Extremely easy & $\square$ Extremely easy \\
\hline
\end{tabular}

2. How smooth is the robotic manipulation?

\begin{tabular}{|l|c|}
\hline Virtually point-constrained joystick interface & Unconstrained, direct-position interface \\
\hline$\square$ Extremely unsmooth & $\square$ Extremely unsmooth \\
$\square$ Unsmooth & $\square$ Unsmooth \\
$\square$ Indifferent & $\square$ Indifferent \\
$\square$ Smooth & $\square$ Smooth \\
$\square$ Extremely smooth & $\square$ Extremely smooth \\
\hline
\end{tabular}

3. How stable is the robotic manipulation?

\begin{tabular}{|l|c|}
\hline Virtually point-constrained joystick interface & Unconstrained, direct-position interface \\
\hline$\square$ Extremely unstable & $\square$ Extremely unstable \\
$\square$ Unstable & $\square$ Unstable \\
$\square$ Indifferent & $\square$ Indifferent \\
$\square$ Stable & $\square$ Stable \\
$\square$ Extremely stable & $\square$ Extremely stable \\
\hline
\end{tabular}

4. How accurate is the robotic manipulation?

\begin{tabular}{|l|c|}
\hline Virtually point-constrained joystick interface & Unconstrained, direct-position interface \\
\hline$\square$ Extremely inaccurate & $\square$ Extremely inaccurate \\
$\square$ Inaccurate & $\square$ Inaccurate \\
$\square$ Indifferent & $\square$ Indifferent \\
$\square$ Accurate & $\square$ Accurate \\
$\square$ Extremely accurate & $\square$ Extremely accurate \\
\hline
\end{tabular}

5. What do you think of the added motion scaling in the control interface?

$\square$ Great-very helpful

$\square$ Good-somewhat helpful

$\square$ Indifferent

$\square$ Not helpful 
6. What is your general impression of the robotic control?

\begin{tabular}{|c|c|c|c|}
\hline \multicolumn{2}{|l|}{ Virtually point-constrained joystick interface } & Unconstrained, direct-position interface \\
\hline Camera Guidance & Tool Manipulation & Camera Guidance & Tool Manipulation \\
\hline$\square$ Awful & $\square$ Awful & $\square$ Awful & $\square$ Awful \\
$\square$ Poor & $\square$ Poor & $\square$ Poor & $\square$ Poor \\
$\square$ Indifferent & $\square$ Indifferent & $\square$ Indifferent & $\square$ Indifferent \\
$\square$ Good & $\square$ Good & $\square$ Good & $\square$ Good \\
$\square$ Wonderful & $\square$ Wonderful & $\square$ Wonderful & $\square$ Wonderful \\
\hline
\end{tabular}

7. Given the choice, which control interface would you use for camera guidance?

$\square$ Virtually point-constrained joystick interface

$\square$ Unconstrained, direct-position interface

8. Given the choice, which control interface would you use for tool manipulation?

$\square$ Virtually point-constrained joystick interface

$\square$ Unconstrained, direct-position interface

\section{Having force feedback in the joystick}

$\square$ significantly hinders your ability to perform surgical tasks

$\square$ slightly hinders your ability to perform surgical tasks

$\square$ doesn't make a difference

$\square$ slightly helps your ability to perform surgical tasks

$\square$ greatly helps your ability to perform surgical tasks

\section{Your speed and overall ability seem to be}

$\square$ greatly increased using the robotic tool as compared to a manual tool

$\square$ slightly increased using the robotic tool as compared to a manual tool

$\square$ unchanged when using the robotic tool as compared to a manual tool

$\square$ slightly lessened using the robotic tool as compared to a manual tool

$\square$ greatly lessened using the robotic tool as compared to a manual tool

11. Would you be interested in using this robotic tool once commercially available?

$\square$ Yes

$\square$ No

$\square$ Maybe 



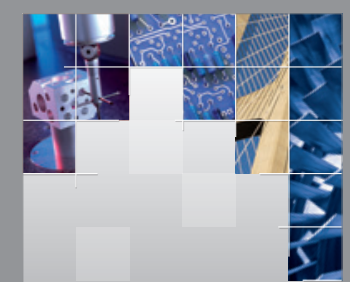

\section{Enfincering}
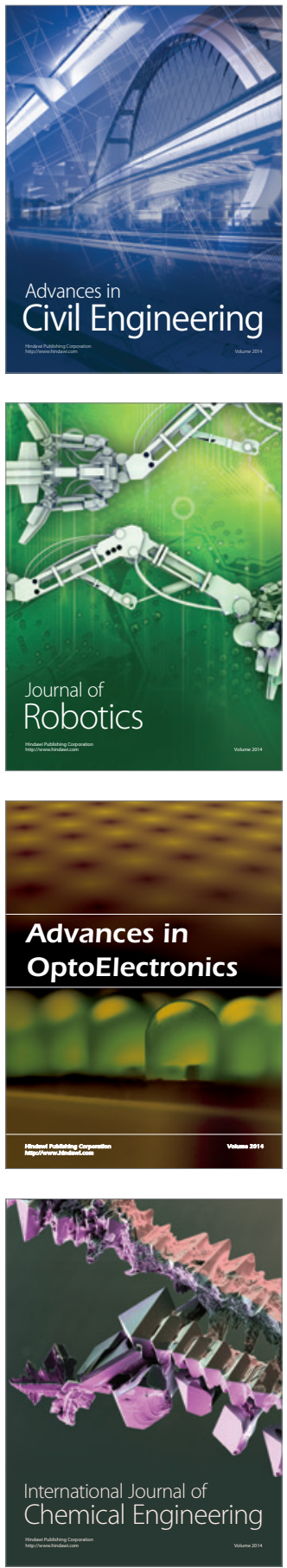

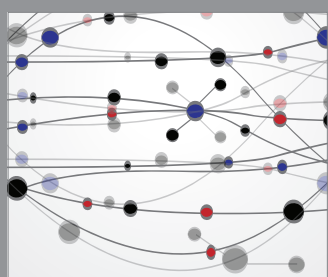

The Scientific World Journal

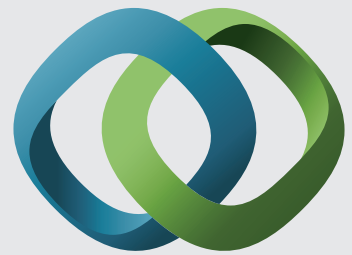

\section{Hindawi}

Submit your manuscripts at

http://www.hindawi.com
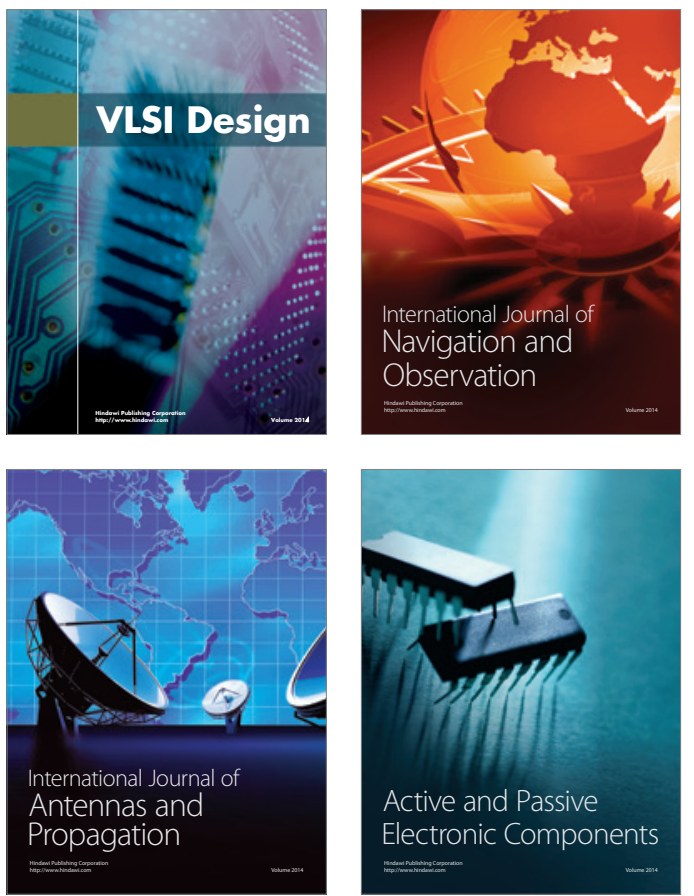
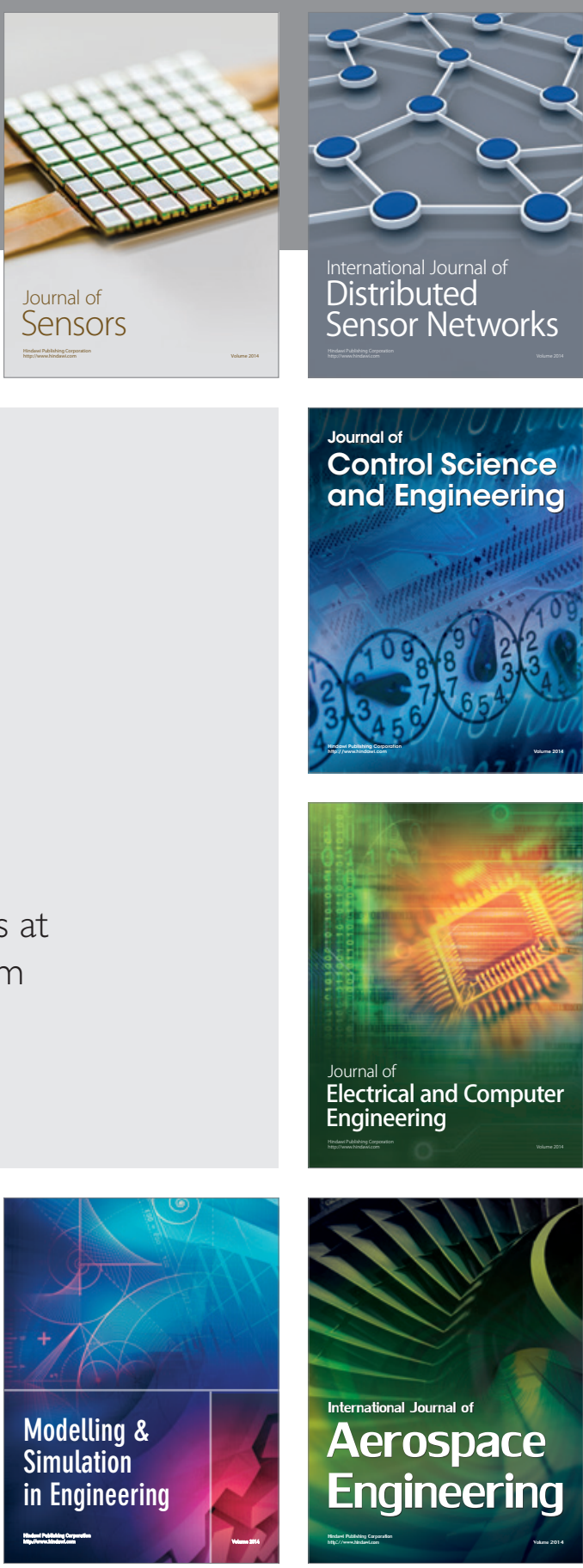

International Journal of

Distributed

Sensor Networks

Journal of

Control Science

and Engineering
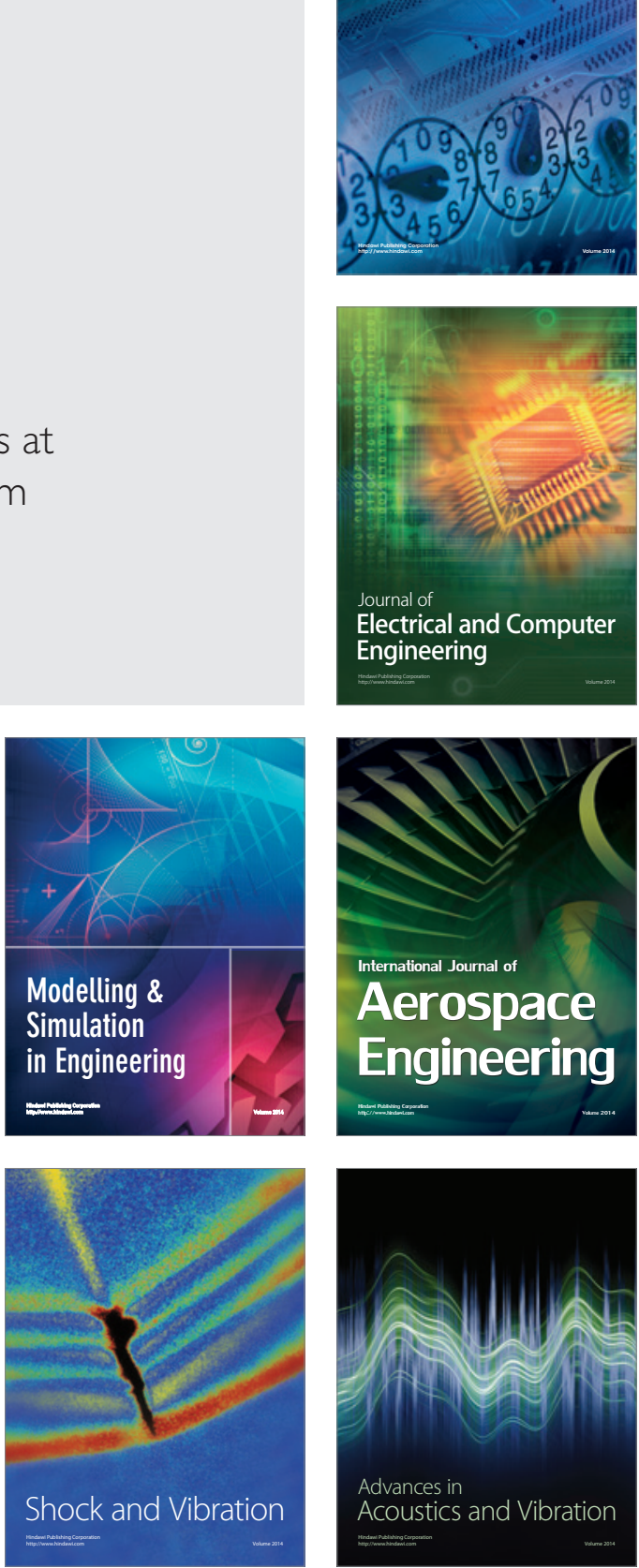